ISSN 1112-9867

\title{
REPLACEMENT OF BARLEY GRAINS AND DEHYDRATED ALFALFA BY SULLA HAY (HEDYSARUM FLEXUOSUM) AND COMMON REED LEAVES (PHRAGMITES AUSTRALIS) IN FATTENING RABBITS DIET
}

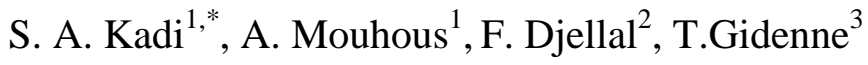 \\ ${ }^{1}$ Département des sciences agronomiques; Université Mouloud MAMMERI de Tizi-Ouzou. \\ ${ }^{2}$ Département d'agronomie, Faculté des sciences de la nature et de la vie, Université Ferhat \\ ABBAS. Sétif-1-. \\ ${ }^{3}$ GenPhySE, Université de Toulouse, INRA, INPT, INP-ENVT, Castanet Tolosan, France \\ Received: 14 Jully 2016 / Accepted: 15 December 2016 / Published online: 01 January 2017
}

\begin{abstract}
Fifty-five Algerian white population rabbits were used to study the effect of the incorporation of Sulla hay (Hedysarum flexuosum) and common Reed leaves (Phragmites australis) in a pelleted feed on the growth of rabbits. At weaning $(35 \mathrm{~d})$, the rabbits were divided in two groups, individually caged and received ad libitum one of the two diets during $42 \mathrm{~d}$. The control diet contained barley, dehydrated alfalfa, soya bean meal and wheat bran. The other diet (RS) was formulated to totally substitute barley and dehydrated alfalfa with Sulla hay and common Reed leaves. Feed intake increased distinctly in the RS group in the second period of fattening (150 vs 126). For the whole fattening period (35-77 d), growth rate was similar in the two groups $(35.4 \mathrm{~g} / \mathrm{d})$ while the feed conversion was higher $(\mathrm{p}<0.01)$ in RS group $(3.86 \mathrm{vs}$ 3.23). This confirmed that using these fibre sources in a pelleted feed was valuable for fattening rabbit.
\end{abstract}

Keywords: Rabbit, Growth performances, Phragmites australis, Hedysarum flexuosum.

Author Correspondence, e-mail: kadisiammar@yahoo.fr

doi: http://dx.doi.org/10.4314/jfas. v9i1.2 


\section{INTRODUCTION}

Le Sulla (Hedysarum flexuosum), légumineuse disponible localement, présente une valeur nutritive appréciable pour les lapins en engraissement : 8,96 MJ/kg MS d'énergie digestible et $71 \mathrm{~g} / \mathrm{kg}$ MS de protéines digestibles selon Kadi et al. [1]. De même, les feuilles de Roseau (Phragmites australis), disponibles en grande quantité et non valorisées, sont une bonne source de fibres pour le lapin en engraissement. Selon Kadi et al. [2], elles contiennent au stade de maturité (en \% de MS) 69\% NDF, 41\% ADF et 11,5\% d'ADL alors que leur apports en ED et PD sont très faibles : apports nuls en ED et $32 \mathrm{~g} / \mathrm{kg}$ MS de protéines digestibles. Après la détermination de la valeur nutritive de ces deux matières premières, l'on se propose d'étudier dans cet essai la possibilité de les associer dans un même aliment granulé pour le lapin en engraissement.

L'objectif de cet essai est donc, d'étudier l'effet de l'incorporation du foin de Sulla (Hedysarum flexuosum) et des feuilles de Roseau (Phragmites australis) dans l'aliment sur les performances zootechniques des lapins en croissance.

\section{MATERIEL ET METHODES}

\subsection{Les aliments expérimentaux}

Le Sulla (Hedysarum flexuosum) a été récolté, au printemps (stade début floraison), au niveau de la région d'Oued-Aissi à Tizi-Ouzou et séché naturellement par étalage au soleil au niveau du CFPA de Mechtras où sont, aussi, récoltées (durant l'été) et séchées les feuilles de Roseau (Phragmites australis). Ces deux matières premières sont ensuite broyées et acheminées à l'UAB SARL "Production locale" sise à Bouzareah (Alger) pour la fabrication des aliments granulés.

Un aliment expérimental "RS" contenant 20\% de foin de Sulla et 20\% de feuilles séchées de Roseau (Tableau 1), en remplacement total de la luzerne et de l'orge, a été formulé pour répondre aux recommandations nutritionnelles des lapins en croissance [3]. Il a été comparé à un aliment témoin $(\mathrm{T})$ classique à base de luzerne et d'orge. Aucun anticoccidien ni antibiotique n'a été rajouté aux deux aliments.

\subsection{Les animaux et déroulement de l'expérimentation}


L'essai est réalisé au niveau du clapier pédagogique du CFPA de Mechtras. Un total de 55 lapereaux de population locale blanche, nés et sevrés au sein d'un clapier privé limitrophe du CFPA de Mechtras, ont été répartis selon le poids vif initial et l'origine de la portée, à raison de 25 pour le lot $\mathrm{T}$ et 30 pour le lot RS. Ils ont été placés dans des cages individuelles entièrement métalliques disposées en flat-deck et alimentés ad libitum avec un des deux aliments, durant 6 semaines. Le poids vif et la consommation alimentaire ont été mesurés chaque semaine. L'abreuvement a été automatique.

\subsection{Analyses chimiques}

Les analyses chimiques des aliments ont été réalisées au niveau de l'INRA (UMR GenPhySE) de Toulouse (France) et ont concerné les parametres suivants : humidité, cendres, matières azotées totales ( $\mathrm{N}$ x 6,25, méthode Dumas, Leco), énergie (Bomb calorimeter method), fibres (NDF, ADF et ADL) selon la méthode séquentielle de Van Soest.

\subsection{Analyses statistique}

Les données obtenues ont été soumises à une analyse de variance (procédure GLM) à l'aide du logiciel SAS (OnlineDoc ${ }^{\circledR}$, SAS Inst., Cary, NC) en prenant en compte le facteur lot.

\section{RESULTATS ET DISCUSSION}

\subsection{Caractéristiques nutritionnelles des aliments}

La composition chimique des aliments (Tableau 1) a été en dessus des valeurs prévues par formulation pour les protéines brutes, mais en deçà des apports en ED attendues (formulation $v s$ équation de prédiction). Pour ce qui est des fibres (NDF, ADF), leur niveau a dépassé les prévisions dans l'aliment T, mais était conforme dans l'aliment RS.

La teneur en minéraux est substantiellement augmentée par le foin de Sulla et les feuilles de roseau, les deux étant riches en minéraux (Tableau 1). L'incorporation du Sulla et des feuilles de roseau conduit logiquement à une augmentation du taux de fibres notamment NDF et ADF. De la même manière, l'incorporation du Sulla et des feuilles de roseau avec, respectivement, des teneurs en EB de 17,2 et 18,5 MJ/kg, a permis de maintenir la teneur en EB au même niveau que dans le témoin malgré l'absence de la luzerne, mais surtout de l'orge. Par contre, les concentrations en énergie digestible, égales au niveau des deux aliments, ne semblent pas 
atteindre les valeurs recommandées pour les lapins en engraissement. En plus du fait que l'estimation de l'ED à partir des tables est inexacte [4], cette situation serait liée, notamment dans l'aliment expérimental, aux apports nuls en énergie des feuilles de roseau comme rapporté par Kadi et al. [2].

Tableau 1. Composition des aliments expérimentaux

\begin{tabular}{|c|c|c|c|c|}
\hline Ingrédients (\%) & Aliment $\mathrm{T}$ & Aliment RS & $\begin{array}{l}\text { Foin de } \\
\text { Sulla }^{*}\end{array}$ & $\begin{array}{l}\text { Feuilles de } \\
\text { Roseau }^{* *}\end{array}$ \\
\hline Foin de Sulla & - & 20 & & \\
\hline Feuilles de Roseau & - & 20 & & \\
\hline Orge & 20 & - & & \\
\hline Luzerne déshydratée & 35 & - & & \\
\hline Tourteau de soja & 13 & 14 & & \\
\hline Son de blé & 30.5 & 44,5 & & \\
\hline Sel & 0,5 & 0,5 & & \\
\hline Premix & 1 & 1 & & \\
\hline \multicolumn{5}{|c|}{ Composition chimique, $\mathrm{g} / \mathrm{kg} M S$} \\
\hline Matière sèche & 906 & 905 & 885 & 93,17 \\
\hline Matières minérales & 117 & 151 & 142 & 130 \\
\hline Protéines brutes & 201 & 212 & 165,7 & 110 \\
\hline $\mathrm{NDF}$ & 360 & 440 & 486,4 & 690 \\
\hline $\mathrm{ADF}$ & 185 & 206 & 345,3 & 410 \\
\hline ADL & 51 & 55 & 90,3 & 115 \\
\hline $\mathrm{EB}, \mathrm{MJ} / \mathrm{kg}$ & 18,29 & 18,38 & 17,02 & 18,25 \\
\hline $\mathrm{ED}, \mathrm{MJ} / \mathrm{kg}$ & $9,59^{\mu}$ & $9,17^{\mu}$ & $8,9^{*}$ & $1,8^{* *}$ \\
\hline $\mathrm{PD}, \mathrm{g} / \mathrm{kg} \quad \#$ & $141^{\#}$ & $151^{\#}$ & $71^{*}$ & $29^{* *}$ \\
\hline $\mathrm{PD} / \mathrm{ED}, \mathrm{g} / \mathrm{MJ}, \mathrm{MS}$ & 14,8 & 16,5 & - & - \\
\hline \multicolumn{5}{|c|}{ * : D'après Kadi et al. [1] ; $* *$ : d'après Kadi et al. [2] } \\
\hline \multicolumn{5}{|c|}{${ }^{\mu}$ estimé par l'équation de De Blas [5]: } \\
\hline \multicolumn{5}{|c|}{$\mathrm{ED} \mathrm{Kcal} / \mathrm{kg} \mathrm{MS}=239 \times(13,30-0,201 \mathrm{ADF}(\% \mathrm{MS})+0,102 \mathrm{MAT}(\% \mathrm{MS})$} \\
\hline \multicolumn{5}{|c|}{ \# estimé par l'équation de Villamide et Fraga [6] : PD $(\mathrm{g} / \mathrm{kg})=-34,67+0.876$ x PB $(\mathrm{g} / \mathrm{kg})$} \\
\hline
\end{tabular}

Les apports protéiques des deux aliments dépassent largement les prévisions ainsi que les recommandations pour les lapins en croissance [3] et qui sont de $15 \%$. Au niveau de l'aliment témoin, cela pourrait s'expliquer par la variation de la composition chimique des matières premières dont la provenance est diverse [7] ; alors que lors de la formulation l'on se base sur les tables disponibles dans la bibliographie. De plus, les deux aliments contiennent des quantités importantes de son de blé (30,5 et 44,5\% respectivement) alors que ce dernier est 
connu pour la forte fluctuation de sa composition chimique selon la composition du blé et les conditions de mouture [8]. En Algérie, Boudouma [9] signale que les caractéristiques chimiques du son de blé produit localement présentent une forte variabilité entre les moulins où ils sont produits, mais également au sein d'un même moulin permettant d'avoir, par exemple, des teneurs en protéines variant de 12,4 à 19,1\% MS. A titre d'exemple, pour étayer cette variation de composition chimique des matières premières utilisées, l'aliment témoin utilisé par Kadi et al. [1] et celui utilisé par Kadi et al. [2] dans l'essai de détermination de la valeur nutritive des feuilles de Roseau, sont composés des mêmes ingrédients et aux mêmes proportions (même formule alimentaire) ; fabriqués au niveau de la même unité d'aliment de bétail ; avec une année d'intervalle, leur composition chimique est sensiblement différente notamment en minéraux, NDF, ADF et protéines avec, respectivement, 44,6;21,0;10,4 et $10,0 \%$ de différence.

Il est à remarquer qu'un rapide calcul théorique combinant la teneur en protéines et la composition centésimale (taux d'incorporation des matières premières) de l'aliment expérimental (RS) fait ressortir la possibilité de se passer totalement du tourteau de soja au profit soit du Sulla soit du son de blé. En effet, incorporé à $14 \%$ le tourteau de soja apporte 6 des 21 points de protéines de cet aliment, 6 points qui sont en excédent. Cet excès des apports en protéines dans les deux aliments conjugués au léger déficit en énergie conduit, logiquement, à rapport PD/ED élevé, qui dépasse largement les récentes recommandations. Selon Carabano et al. [10], un rapport PD/ED de 9,5 est correct et ne paraît pas diminuer les performances de croissance si l'apport en acides aminés est correct.

\subsection{Croissance et consommation alimentaire}

Au cours de l'expérimentation, 3 lapins ont été perdus dans le lot ayant consommé l'aliment RS alors qu'aucune mortalité n'a été enregistrée dans le lot témoin. Dans le lot RS, les pertes sont enregistrées à la première semaine ce qui peut être attribué au stress causé par le sevrage, au changement de clapier et l'installation en cages [11].

Durant la première période d'engraissement (35-56 j), les lapins ont évolué de la même manière dans les deux lots avec, comme observé généralement, de très bonnes performances (Tableau 2) : une croissance de $38 \mathrm{~g} / \mathrm{j}$ et un indice de consommation de 2,8 en moyenne. 
Durant la deuxième période (56-77 j), les performances des lapins ayant consommé l'aliment RS se sont sensiblement détériorées. En effet, si la vitesse de croissance a été maintenue par rapport au lot témoin (près de $32 \mathrm{~g} / \mathrm{j}$ en moyenne), ce n'est pas le cas des quantités consommées qui ont été beaucoup plus importantes (126 vs $150 \mathrm{~g} / \mathrm{j}, \quad \mathrm{P}<0001)$. En conséquence, l'indice de consommation s'est sensiblement ( $\mathrm{p}=0,021)$ détérioré $(4,02$ vs 4,94). Cette situation serait en relation avec les apports énergétiques qui seraient insuffisants surtout dans l'aliment RS à cause des très faibles apports des feuilles de roseau.

Pour ce qui est de la période globale (35-77j), les deux aliments ont permis aux lapins de réaliser des poids vifs supérieurs à $2 \mathrm{~kg}$ en 6 semaines d'engraissement, avec un léger avantage à la limite de la signification pour l'aliment RS : 2,06 kg et 2, $22 \mathrm{~kg}$ respectivement $(\mathrm{p}=0,055)$. La vitesse de croissance n'a pas été influencée par le type d'aliment et a été en moyenne de 35,4 g/j malgré l'excès en protéines. Selon Gidenne et al. [12], un excès de protéines n'affecte pas la croissance, mais peut favoriser l'apparition de diarrhée, ce qui n'a pas été le cas dans cet essai.

Tableau 2. Vitesse de croissance et consommation alimentaire

\begin{tabular}{lcccc}
\hline & Aliment T & Aliment RS & SEM & $P$ \\
\hline $\mathrm{n}^{1}$ & 25 & 27 & & \\
Période 35-56 j & & & & \\
$\quad$ Poids vif à 35 j, g & 601 & 745 & 69 & 0,15 \\
Poids vif à 56 j, g & 1411 & 1543 & 72 & 0,20 \\
Gain de poids, g/j & 38,6 & 37,3 & 0,83 & 0,30 \\
Consommation alimentaire, g/j & 101,0 & 113,8 & 5,1 & 0,083 \\
Indice de consommation, g/g & 2,63 & 2,88 & 0,12 & 0,16 \\
Période 56-77 j & & & & \\
Poids vif à 77 d, g & 2058 & 2220 & 58,32 & 0,055 \\
Gain de poids, g/j & 31,7 & 32,2 & 1,17 & 0,76 \\
Consommation alimentaire, g/j & 126,4 & 150,0 & 3,68 & $<0,001$ \\
Indice de consommation, g/g & 4,02 & 4,94 & 0,27 & 0,021 \\
Période 35-77 j & & & & \\
Gain de poids, g/j & 35,3 & 35,5 & 0,69 & 0,80 \\
Consommation alimentaire, g/j & 113,9 & 133,8 & 3,88 & $<0,001$ \\
Indice de consommation, g/g & 3,23 & 3,86 & 0,15 & 0,0093 \\
\hline$\quad$ n: nombre de lapins à la fin de la période expérimentale & &
\end{tabular}

L'incorporation du foin de Sulla et des feuilles de Roseau n'a donc pas affecté la croissance 
des lapins. Par contre, le maintien de la vitesse de croissance au même niveau dans les deux lots l'a été au prix d'une élévation de $25 \%$ de l'indice de consommation ( $\mathrm{p}=0,0093)$ au niveau du lot RS, du fait de la plus grande consommation de cet aliment par rapport au témoin (134 vs $114 \mathrm{~g} / \mathrm{j}$ respectivement, $\mathrm{p}<0,001)$.

La vitesse de croissance obtenue dans cet essai $(35,4 \mathrm{~g} / \mathrm{j})$ est dans la moyenne de ce que l'on a enregistré sur cette population de lapins et avec des aliments contenant soit du foin de Sulla soit des feuilles de Roseau. La meilleure vitesse de croissance $(38 \mathrm{~g} / \mathrm{j})$ a été enregistrée par Kadi et al. [1] avec un aliment contenant $15 \%$ de foin de Sulla. Quant à la plus faible vitesse de croissance, elle a été de 31,1 g/j enregistré par Kadi et al. [2], sur la même population de lapin, avec un aliment contenant 30\% de feuilles de Roseau. Sur cette même population de lapin, la meilleure vitesse de croissance enregistrée par Lounaouci-Ouyed et al. [13] a été de 32,05 g/j avec l'aliment témoin. Comparée aux résultats obtenus avec des lapins de population locale colorée, la vitesse de croissance enregistrée ici $(35,4 \mathrm{~g} / \mathrm{j})$ est largement supérieure à la meilleure valeur $(30,5 \mathrm{~g} / \mathrm{j})$ signalée par Berchiche et al. [14] et à celle $(28 \mathrm{~g} / \mathrm{j})$ raportée par Lakabi et al. [15] ou à celle (23,6 g/j) enregistrée par Guemour et al. [16].

Les indices de consommation enregistrés sont en deçà des normes dans les deux lots même si celui enregistré dans le lot témoin est conforme à ce que l'on a l'habitude de trouver dans les mêmes conditions $[1,13,15]$. Cependant, l'aliment RS est le moins efficacement transformé (3,86 vs 3,23; p=0,0093). Cette situation est logiquement reliée aux consommations, la vitesse de croissance étant hors de cause, qui sont plus importantes dans ce lot (133,8 vs 113,9 $\mathrm{g} / \mathrm{j} ; \mathrm{P}=0,0006)$ et qui ont nettement augmenté durant la deuxième période (56-77j) d'engraissement (150 vs 126,4 g/j respectivement pour l'aliment RS et $\mathrm{T}$; $\mathrm{p}<0,0001$ ). Résultat de cette surconsommation durant la deuxième période, l'indice de consommation s'est nettement détérioré (4,94 vs 4,02 respectivement pour l'aliment RS et T; $p=0,021)$ alors qu'il a été en moyenne de 2,8 durant la première période et ce même si une détérioration est inévitable avec l'augmentation de l'âge des lapins du sevrage à l'âge d'abattage [17]. La concentration en ED des aliments serait la cause de cette surconsommation. En effet, les apports énergétiques seraient en deçà des recommandations dans les deux aliments, mais surtout dans l'aliment RS. Selon Xiccato et Trocino [17], la concentration en ED explique en 
grande partie la variabilité des quantités consommées et de l'indice de consommation. Selon ces auteurs, une augmentation de l'ED de l'aliment de $1 \mathrm{MJ} / \mathrm{kg}$ diminue la consommation de $12 \mathrm{~g} / \mathrm{j}$ et l'indice de consommation de 0,29 point, diminution de l'indice de consommation que Maertens [18] estime à 0,15-0,20 points. Par contre, Gidenne et Lebas [19] signalent que le niveau d’ingestion, par conséquent l'indice de consommation, est mieux corrélé avec la concentration en fibres de l'aliment, qu'avec la concentration en ED.

La valeur de l'indice de consommation enregistré dans le lot RS est la même $(3,8)$ que celle enregistrée par Kadi et al. [20] avec un aliment composé de $61 \%$ de son de blé, $20 \%$ de grignon d'olives et $18 \%$ de tourteau de soja. Cette valeur $(3,8)$ est, par contre, meilleure que celle rapportée par Berchiche et al. [14] avec un aliment pourtant témoin (4,5), mais nettement meilleure que celle signalée par Mefti-Korteby et al. [21] et qui a atteint 5,6 à 70 jours et même 7,1 à 91 jours.

\section{CONCLUSION}

L'incorporation du foin de Sulla (Hedysarum flexuosum) et des feuilles de Roseau (Phragmites australis) dans l'aliment des lapins en croissance, en remplacement de l'orge et de la luzerne déshydratée, a permis de maintenir la vitesse de croissance au même niveau que celle du lot témoin $(35,4 \mathrm{~g} / \mathrm{j})$ avec, cependant, une élévation significative de l'indice de consommation.

Il est confirmé que l'utilisation de ces deux fourrages dans l'aliment granulé pour lapins en croissance est intéressante.

Cependant, la composition de l'aliment contenant ces deux matières premières n'étant pas tout à fait équilibrée, d'autres investigations, avec des aliments parfaitement équilibrés, mais aussi avec des taux d'incorporation variés, sont nécessaires.

\section{REFERENCES}

[1] Kadi S A, Guermah H, Bannelier C, Berchiche M, Gidenne T. World Rabbit Sci., 2011, 19, 151-159,

doi:10.4995/wrs.2011.848 
[2] Kadi S A, Ouendi M, Slimani M, Selmani K, Bannelier C, Berchiche M and Gidenne T. Nutritive value of common reed (Phragmites australis) leaves for rabbits. 10 th World Rabbit Congress, 2012, 513-517

[3] De Blas C., Mateos, G.G. Feed formulation. In: C. De Blas, \& J. Wiseman (Eds.), Nutrition of the rabbit, CABI, 2010, pp.222-232.

[4] Fernández-Carmona J, Soriano J, Pascual J J and Cervera C. The prediction of nutritive value of rabbit diets from tables of feed composition. 8 ${ }_{\text {}}$ World Rabbit Congress, 2004, Puebla, 818-823.

[5] De Blas C. Nutrition et alimentation du lapin. 1994. Institut agronomique méditerranéen de Saragosse, 10 février au 25 mars.

[6] Villamide MJ, Fraga MJ. Anim. Feed Sci. Technol. 1998, 70: 221-224.

doi:10.1016/S0377-8401(97)00079-5

[7] Bastianelli D, Epaku OR, Bonnal L, Grimaud P. Qualité des matières premières : résultats d'une étude en Afrique de l'Est. Perspectives pour la gestion de la variabilité des matières premières. RASPA, 2009, 7 (S) :33-40.

[8] Blas E, Fernandez-Carmona J, Cervera C, Pascual JJ. Anim. Feed Sci. Technol. 2012, 88, 239-251

doi: 10.1016/S0377-8401(00)00212-1

[9] Boudouma D. Composition chimique du son de blé dur produit par les moulins industriels algériens. Livestock Research for Rural Development, 2009, 21 (10).

[10] Carabaño R, Villamide MJ, García J, Nicodemus N, Llorente A, Chamorro S, Menoyo D, García-Rebollar P, García-Ruiz AI, De Blas JC. World Rabbit Sci. 2009, 17: 1-14 doi: http://dx.doi.org/10.4995/wrs.2009.664

[11] De Blas J C, Chamorro S, García-Alonso J, García-Rebollar P, García-Ruiz A I , Gómez-Conde M S, Menoyo D, Nicodemus N, Romero C, Carabano R. Anim. Feed Sci. Technol. 2012, 173, 102-110.

doi:10.1016/j.anifeedsci.2011.12.016

[12] Gidenne T., García J., Lebas F., Licois D. Nutrition and Feeding Strategy: Interactions with Pathology. In C. De Blas \& J. Wiseman (Eds.), Nutrition of the rabbit, CABI, 2010, 
pp.179-199.

[13] Lounaouci-Ouyed G, Lakabi-Ioualitene D, Berchiche M, Lebas F. 9th World Rabbit Congress, 2008, Verona, 123-728.

[14] Berchiche M, Kadi S A, Lebas F. 7th World Rabbit Congress, 2000, Valencia, 119-124.

[15] Lakabi D, Lounaouci G, Berchiche M, Lebas F, Lamothe L. World Rabbit Sci., 2008. 16: 99-106.

doi: http://dx.doi.org/10.4995/wrs.2008.632

[16] Guemour D, Bannelier C, Della A, Gidenne T. World Rabbit Sci., 2010. 18:17-25.

doi: http://dx.doi.org/10.4995/wrs.2010.18.03

[17] Xiccato G, Trocino A. 6th Inter. Con. on Rabbit Prod. in Hot Clim, 2010, Egypt, 1-18.

[18] Maertens L. 2010. Feeding Systems for Intensive Production. In C. De Blas \& J. Wiseman (Eds.), Nutrition of the rabbit, CABI, 2010, pp.253-266.

[19] Gidenne T, Lebas F. 11èmes Journées Recherche Cunicole, 2005, Paris, 183-196.

[20] Kadi SA, Belaidi-Gater N, Chebat F. 8th World Rabbit Congress, 2004, Puebla, 1202-1207.

[21] Mefti-Korteby H, Kaidi R, Sid S, Daoudi O. European Journal of Scientific Research, 2010.40 (1) $132-143$.

\section{How to cite this article:}

Kadi S.A, Mouhous A, Djellal F, Gidenne T. Replacement of barley grains and dehydrated alfalfa by sulla hay (hedysarum flexuosum) and common reed leaves (phragmites australis) in fattening rabbits diet. J. Fundam. Appl. Sci., 2017, 9(1), 13-22. 